\title{
Embedded spectroscopic fiber sensor for on-line arc-welding analysis
}

\author{
Jesús Mirapeix, ${ }^{\star}$ Adolfo Cobo, Antonio Quintela, and José-Miguel López-Higuera \\ Grupo de Ingeniería Fotónica-Universidad de Cantabria E.T.S.I.I. y Telecomunicación-Dpto. TEISA Avda. \\ Los Castros s/n-39005 Santander, Spain \\ ${ }^{*}$ Corresponding author: mirapeixjm@unican.es
}

Received 20 December 2006; revised 26 February 2007; accepted 27 February 2007; posted 2 March 2007 (Doc. ID 78246); published 15 May 2007

\begin{abstract}
A new fiber sensor system designed for spectroscopic analysis and on-line quality assurance of arcwelding processes is presented here. Although several different approaches have been considered for the optical capture of plasma emission in arc-welding processes, they tend to be invasive and make use of optical devices such as collimators or photodiodes. The solution proposed here is based on the arrangement of an optical fiber, which is used at the same time as the optical capturing device and also to deliver the optical information to a spectrometer, embedded within an arc-welding torch. It will be demonstrated that, by using the shielding gas as a protection for the fiber end, the plasma light emission is efficiently collected, forming a sensor system completely transparent and noninvasive for the welding operator. The feasibility of the proposed sensor designed to be used as the input optics of a welding quality-assurance system based on plasma spectroscopy will be demonstrated by means of several welding tests. (C) 2007 Optical Society of America
\end{abstract}

OCIS codes: $\quad 060.2370,300.2140,120.4630,350.3850$.

\section{Introduction}

On-line quality assurance of arc and laser welding processes has been an important research topic for years, and an optimal solution is still to be found. The lack of a theoretical foundation able to effectively model these welding processes [1], mainly due to the inherent complexity of the physics involved, has forced the use of off-line methods (both destructive and non-destructive) to analyze the quality of the welds. In some particular scenarios, like aeronautics or nuclear, the avoidance of possible weld defects is critical, and great efforts are devoted to ensure a specific final quality, typically by using penetrant liquids, magnetic particles, X-rays or macrographs.

Obviously, these off-line quality assurance techniques involve a cost in terms of productivity, which could be minimized by using real-time, on-line monitor and control systems. Several techniques have been proposed to perform an on-line control of welding processes, from the analysis of the plasma acoustic

0003-6935/07/163215-06\$15.00/0

(C) 2007 Optical Society of America emission [2,3], to solutions based on infrared thermography $[4,5]$, or optical approaches typically by means of plasma spectroscopy $[6,7,8]$.

Although plasma spectroscopy has proved to be a promising technique, the development of an optimal on-line welding sensor and control system is still an active area of research. It has been demonstrated that, by performing a spectroscopic analysis of the plasma radiation in the ultraviolet and visible bands, some spectroscopic parameters can be directly correlated with the quality of the resulting welds [6,7]. In addition, the inherent immunity of optical systems to electromagnetic interferences (EMI), which can be a serious problem for the electrical and acoustic solutions, and the wide variety of possible optical sensor setups (remote photodiodes [9] and optical fiber capturing [10]) are some of the advantages of these optical methods.

Despite this diversity regarding the input optics alternatives, the arrangement of optical devices such as collimators in the vicinity of the welding torch can be a drawback in terms of invasiveness. On the other hand, in particular welding scenarios, like processes where complex shapes are considered, the solution to 
the input optics disposition is not trivial. In addition, problems such as heating and interference of weld pool projections with the input optics must be also taken into consideration.

In this paper, a new fiber-sensor approach to the problem of capturing the plasma light radiation during arc welding processes is presented. An optical fiber embedded into a TIG torch is used as the optical sensor of an arc-welding quality assurance technique based on plasma spectroscopy. Several tests performed in the laboratory will show the feasibility of the proposed system.

\section{Plasma Spectroscopy}

The basis of the use of plasma spectroscopy in welding quality assurance lies in the known correlation that exists between the plasma electronic temperature $T_{e}$ profile and its associated weld quality. Consequently, if perturbations are to be found along the $T_{e}$ profile, it will imply that weld flaws will also appear.

The plasma electronic temperature can be determined by using the technique commonly known as Boltzmann-plot, which is derived from the Boltzmann equation [11]:

$$
N_{m}=\frac{N}{Z} g_{m} \exp \left(\frac{-E_{m}}{\kappa T_{e}}\right)
$$

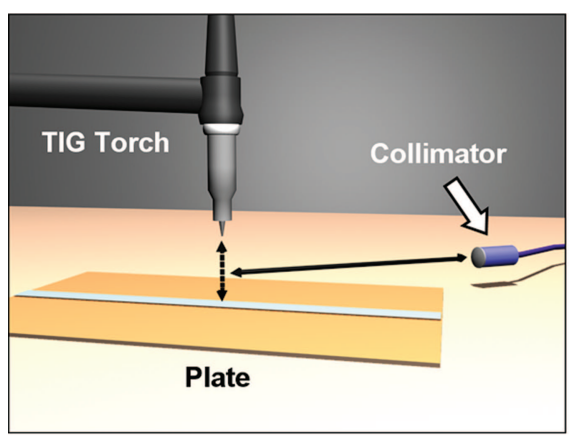

a) Input optics with collimator where $N$ is the population density of the state $m, Z$ the partition function, $g_{m}$ the statistical weight, $E_{m}$ the upper level energy, $\kappa$ the Boltzmann constant and $T_{e}$ the plasma electronic temperature. Considering also the expression relating the intensity of a given emission line to the population density of the upper level $\mathrm{N}_{\mathrm{m}}$,

$$
I_{m n}=N_{m} A_{m n} h \gamma_{m n}
$$

where $A_{m n}$ is the transition probability, and $h \gamma_{m}$ the energy of that transition; then the expression for the so-called Boltzmann-plot can be determined by combining Equations (1) and (2):

$$
\ln \left(\frac{I_{m n} \lambda_{m n}}{A_{m n} g_{m}}\right)=\ln \left(\frac{h c N}{Z}\right)-\frac{E_{m}}{k T_{e}} .
$$

It is worth noting that $T_{e}$ can be calculated with Equation (3), as the plot resulting from using various lines from the same atomic species in the same ionization stage, representing the left-hand side of Equation (3) versus $E_{m}$, has a slope inversely proportional to $T_{e}$. Equation (3) can be used to obtain $T_{e}$ when the plasma is in local thermodynamic equilibrium (LTE) [12], condition that is assumed to be valid when

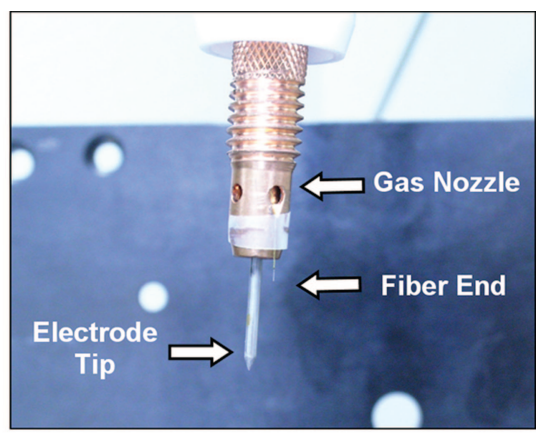

b) Embedded optical fiber

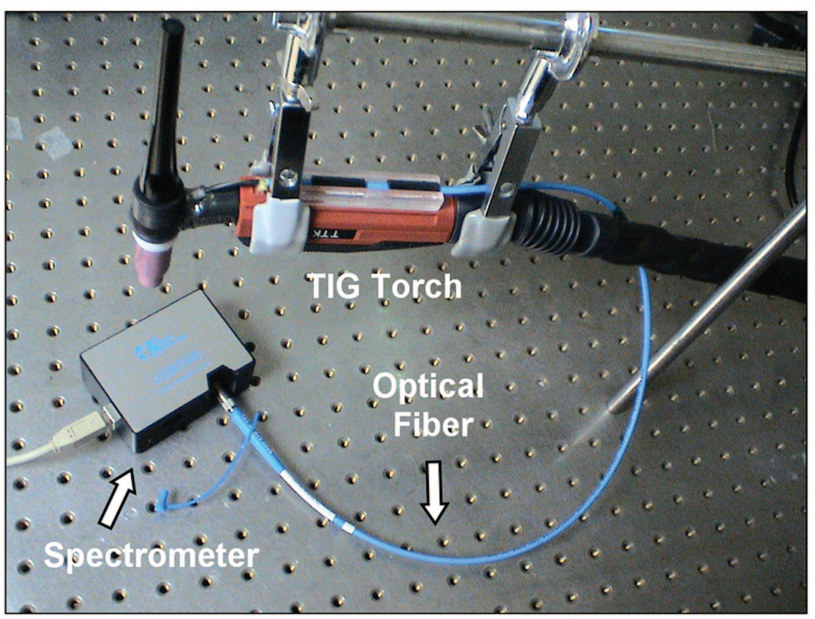

c) TIG torch and fiber sensor setup

Fig. 1. (Color online) Input optics with collimator scheme and image of the fiber embedded into the TIG torch. 


$$
N_{e} \geq 1.6 \times 10^{12} T_{e}^{1 / 2}(\Delta E)^{3},
$$

where $N_{e}$ is the electronic density and $\Delta E$ is the largest energy gap in the atomic energy level system. The criterion expressed in Equation (2) can be obtained by considering that a necessary condition for LTE is that the collision rate has to exceed the spontaneous emission by a factor of ten. The assumption of LTE, which implies that the different particles within the plasma have Maxwellian energy distributions, is traditionally accepted for arc welding plasmas, like those generated during TIG welding processes.

\section{Experimental Issues}

The input optics of the sensor systems designed to capture the light radiated from the plasma formed during the welding process are typically based on the use of a collimator, in addition to an optical fiber and a CCD spectrometer. Although the collimator is a good solution to couple the light radiation into the optical fiber, its dimensions and the necessity to be located centimetres away from the plasma axis are a serious drawback, especially when considering industrial welding processes.

Although some systems whit the input optics attached to the welding torch have been proposed [13], the situation of the collimator outside the torch can be a problem in some scenarios, in terms of operator disturbances or when complex shapes are to be welded.

It is known that the arc-welding process exhibits physical properties that are extremely aggressive to almost any kind of sensor placed just centimetres away from the electrode tip, like strong electromagnetic interference or very high temperatures. The optical fiber is an optimal sensor solution in terms of immunity to electromagnetic interferences, and the use of one of the welding nozzle shielding gas exits to guide the fiber end would allow to reduce the effect of the high temperatures to be found in the vicinity of the welding arc axis. In addition to the cooling effect upon the fiber, the shielding gas flow would also keep possible weld pool projections from colliding with the fiber tip, or from interfering in the light capture process.

A scheme displaying the input optics solutions mentioned above is presented in Figure 1. In Figure 1(a), a scheme of the input optics arrangement considering the use of a collimator is depicted. The collimator should be focused on the axis formed between the electrode tip and the plate to capture the plasma radiation. An image of the proposed sensor is shown in Figure 1(b), where an optical fiber has been embedded within the TIG torch. The ceramic protection of the torch has been removed to enable the view of the fiber end.

The complete setup used to acquire the plasma spectra is presented in Figure 1(c), where the optical fiber, a P50-UVVIS (Ocean Optics), of $50 \mu \mathrm{m}$ core diameter and one meter length, is guided through the torch and attached to a 2048-pixel CCD spectrometer (Ocean Optics USB2000).
Once the fiber was embedded within the torch, it was necessary to validate its ability to both capture the plasma spectra and to resist the arc-welding environment. Several arc-welding tests were performed with a TIG-welding system formed by a "Kemppi Mastertig 2200" power source and a high-precision positioning system, with a controller (Newport MM4005) and two linear stages (Newport MTM100PP1), with a resolution of $1 \mu \mathrm{m}$ in both axes. This positioning system was employed to move the AISI-314 plates used for the tests. In addition, Argon was used as shielding gas (with a flow rate of $12 \mathrm{~L} / \mathrm{min}$ ) and Tungsten electrodes of $1.6 \mathrm{~mm}$ diameter were chosen. The distance between the electrode tip and the plates was $1 \mathrm{~mm}$.

The effect of the welding process on the fiber embedded within the welding torch can be observed in Figure 2. In Figure 2(a) the $50 \mu \mathrm{m}$ core fiber men-

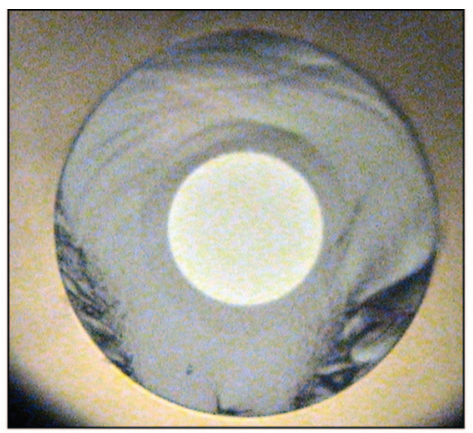

a) Fiber before welding

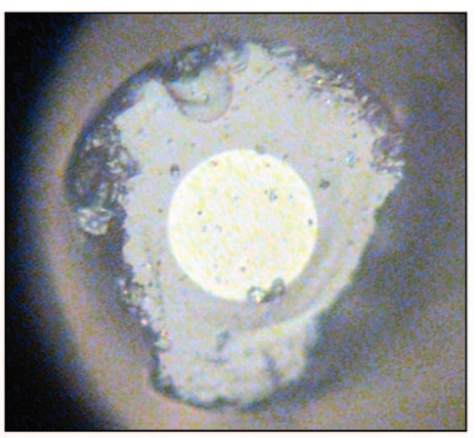

b) Fiber after 10 welding tests

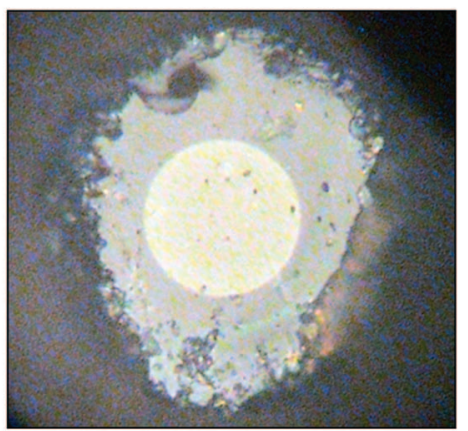

c) Fiber after $\mathbf{3 0}$ welding tests

Fig. 2. (Color online) Optical fibers used as embedded sensor within the welding torch. 
tioned above is shown before having been used as the embedded sensor. In Figures 2(b) and (c) the fiber end after 10 and 30 welding tests is shown. In the case of Figure 2(b) welding currents up to $70 \mathrm{~A}$ were used, while in Figure 2(c) the maximum current was $100 \mathrm{~A}$. It is worth noting that the duration of each welding test was approximately 8 seconds, resulting in total welding times of 80 and 240 seconds for Figures 2(b) and (c) respectively. Although it is clear that some degradation appears in the fiber cladding, the fiber core remains almost unaffected by the welding process, what enables the capture of the plasma radiation. It should be mentioned that the polyimide protection of the fiber was removed by means of the electric arc of a fussion splicer (Ericsson FSU 995), and that the fiber itself was considerably manipulated, what could have affected the fiber tip. In this regard, the defects located at the bottom of the fiber end (left and right sides), could have been provoked

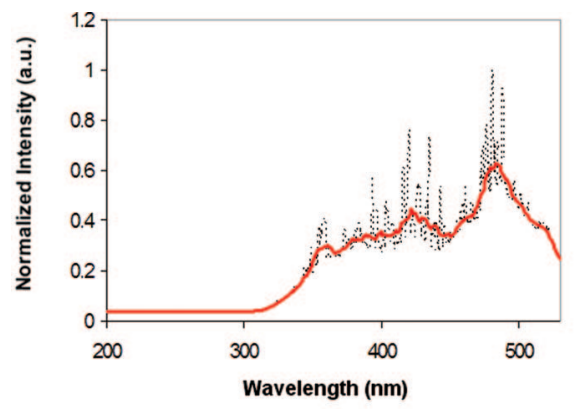

a) Plasma spectrum with collimator

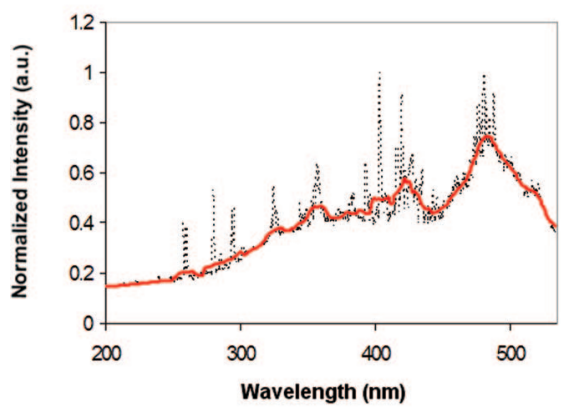

b) Plasma spectrum with embedded fiber (after 10 tests)

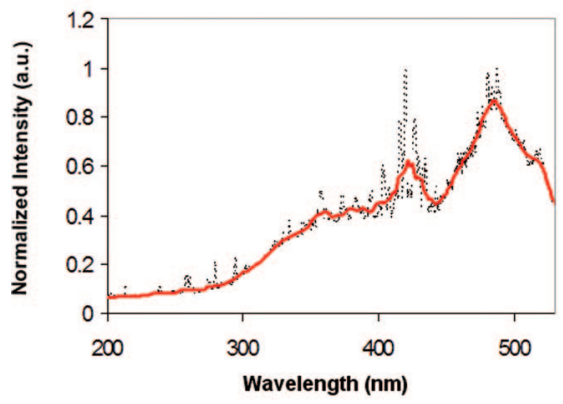

c) Plasma spectrum with embedded fiber (after 30 tests)

Fig. 3. (Color online) Spectra captured with collimator and embedded fiber. by the mechanical stress performed on the fiber when it was guided through the TIG torch.

Examples of spectra captured with the embedded fiber sensor are shown in Figure 3. The captured spectra are depicted with a dotted-line, and the background signal (obtained by means of a moving average) is also represented to facilitate the comparison among spectra. In Figure 3(a) a spectrum captured by means of the input optics scheme of Figure 1(a) is shown. The spectra presented in Figures 3(b) and (c) were obtained with the embedded fiber sensor, and it can be seen that the resulting spectra are quite similar. It is worth noting that, in the case of Figure 3(a), the lack of spectral response in the wavelengths between 200 and approximately $330 \mathrm{~nm}$ is due to the attenuation of the optical fiber in the UV region, given that in this case the length of the fiber used was 2 meters, and the length of the embedded fiber was just 1 meter. In addition, it is worth noting that there is no substantial deterioration in the fiber spectral response after several welding tests, as can be observed by comparing Figures 3(b) and (c). However, a wavelength-dependent loss appears in the fiber in the UV region that can be better appreciated in Figure 4 .

To verify the spectral response of the fiber after several welding tests, a white source was used to record the transmission spectrum of the embedded fiber. A DH-2000 Deuterium Tungsten light source was employed to obtain the data depicted in Figure 2. The Deuterium source was selected, as it provides enough light in the spectral range between 200 and $400 \mathrm{~nm}$. In Figure 4(a) the response of the fiber before and after 50 welding tests (with a total welding time

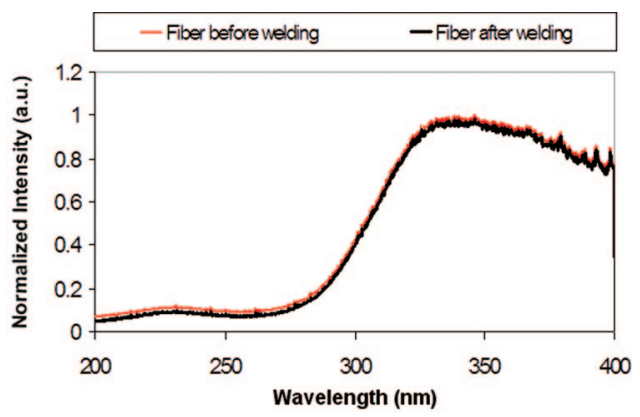

a) Fiber after 50 welding tests (70 A)

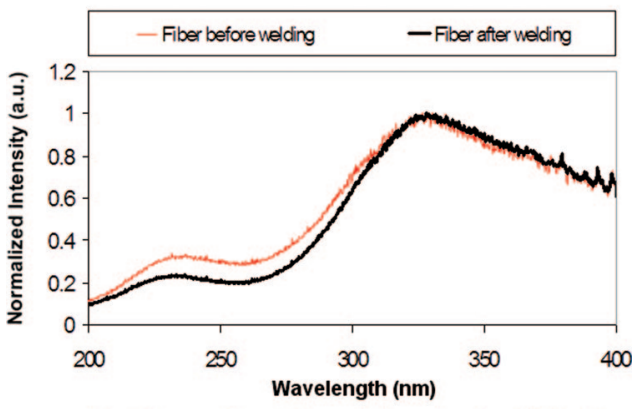

b) Fiber after $\mathbf{3 0}$ welding tests (100 A)

Fig. 4. (Color online) Spectral response of the fiber before and after several welding tests. 


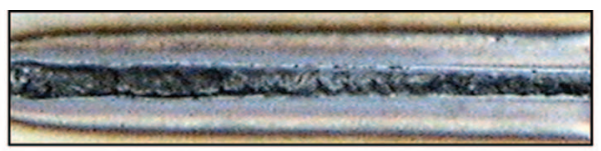

a) Correct weld

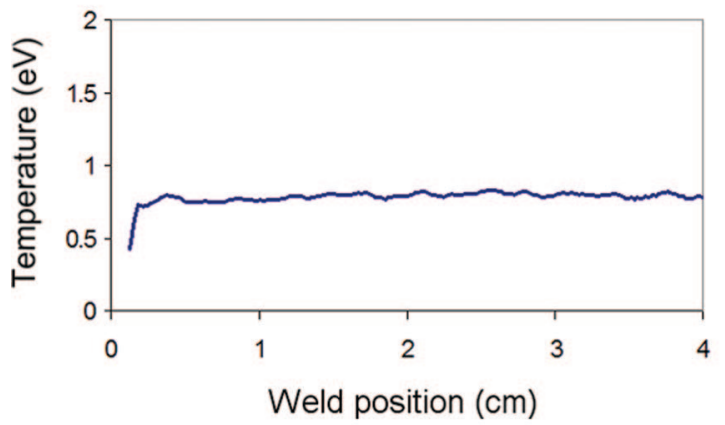

b) Electronic temperature

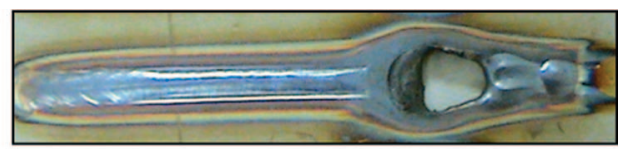

c) Weld with defect

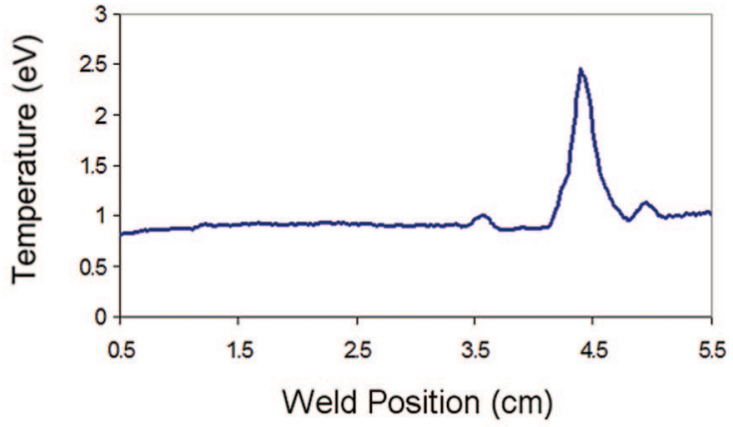

d) Electronic temperature

Fig. 5. (Color online) Examples of weld defect detection by means of the embedded fiber and plasma spectroscopy.

of approximately 400 seconds) is presented, with welding currents up to $70 \mathrm{~A}$. In this case there is no clear deterioration of the optical fiber spectral response, but in Figure 4(b) some attenuation appears in the UV-region (between 200 and $300 \mathrm{~nm}$ ). These additional losses in the UV-region are provoked by the intense radiation in the UV region generated during the welding process (welding currents up to $100 \mathrm{~A}$ were used in Figure 4(b)). This phenomenon, commonly referred to as solarization, is believed to be associated with the formation of color centers $[14,15]$. It is worth noting that the spectral characterization of the fibers is slightly different in Figures 4(a) and (b), because an additional fiber (2 meter length, identical to the one used in the experiments) was used in the characterization for 4 (a).

\section{Spectroscopic Analysis}

The final purpose of the proposed embedded fiber sensor is its implementation within a quality assurance system based on plasma spectroscopy. As mentioned in Section 2, there exists a correlation between the plasma electronic temperature and the quality of the resulting welds, and the $T_{e}$ profiles can be determined using Equation (3).

Several welding tests with the TIG welding setup described in Section 3 were performed to check the

Table 1. Argon Emission Lines Spectroscopic Data

\begin{tabular}{ccccccc}
\hline$\lambda(\mathrm{nm})$ & $\mathrm{E}_{\mathrm{i}}(\mathrm{eV})$ & $\mathrm{E}_{\mathrm{m}}(\mathrm{eV})$ & $\mathrm{g}_{\mathrm{m}}$ & $\begin{array}{c}\mathrm{A}_{\mathrm{mi}} \\
\left(10^{8} \mathrm{~s}^{-1}\right)\end{array}$ & Rel. Int. & $\Delta \mathrm{A}(\%)$ \\
\hline 458.9898 & 18.42655 & 21.12704 & 6 & 0.664 & 400 & 3 \\
460.9567 & 18.45412 & 21.14308 & 8 & 0.789 & 550 & 3 \\
473.5905 & 16.64386 & 19.26109 & 4 & 0.580 & 300 & 3 \\
476.4864 & 17.26583 & 19.86716 & 4 & 0.640 & 800 & 10 \\
480.6020 & 16.64386 & 19.22290 & 6 & 0.780 & 550 & 3 \\
484.7809 & 16.74853 & 19.30535 & 2 & 0.849 & 150 & 10 \\
487.9863 & 17.14003 & 19.68005 & 6 & 0.823 & 800 & 3 \\
\hline
\end{tabular}

validity of the proposed embedded fiber sensor. In Figure 5 two examples showing the ability of the fiber sensor to effectively collect the plasma light radiation to be analyzed by means of plasma spectroscopy are presented. Figure 5(a) depicts a weld where no visible defects are to be found. A constant welding current of $50 \mathrm{~A}$ on a $1 \mathrm{~mm}$ plate was used. The $T_{e}$ profile of Figure 5(b) was determined using Equation (2) with 5 Ar II emission lines located within the spectral range from 474 to $488 \mathrm{~nm}$. The absence of clear perturbations in the $T_{e}$ profile indicates that no defects are expected. On the contrary, the weld shown in Figure 5(c) exhibits a clear defect provoked by a discontinuity in the thickness $(2 \mathrm{~mm})$ of the stainlesssteel plate. A $1 \mathrm{~mm}$ depth incision orthogonal to the weld direction was performed, and this caused the appearance of the void in $x \approx 4 \mathrm{~cm}$, with a constant welding current of $65 \mathrm{~A}$. The associated $T_{e}$ profile (calculated in this case with $7 \mathrm{Ar}$ II lines between 459 and $488 \mathrm{~nm}$ ) presented in Figure 5(d) allows the detection of the mentioned defect by exhibiting a distinguishable perturbation at that weld position, remaining $T_{e}$ constant around $0.85 \mathrm{eV}$ for the rest of the seam. The spectroscopic data of the Ar II emission lines considered is presented in Table 1.

\section{Conclusions}

In this paper a new fiber sensor solution designed to be used for on-line arc-welding process quality assurance by means of spectroscopic analysis has been presented. The fiber sensor, embedded within a TIG welding torch, is a highly non-invasive approach to the problem of effectively collecting the plasma light radiation in arc-welding processes. The use of one of the TIG torch shielding gas nozzle exits to guide the fiber end has a cooling effect upon the fiber, also allowing to prevent possible weld pool projections from interfering with the light capturing process. It has been demonstrated that, although the fiber suf- 
fers some external deterioration due to the welding process, the fiber core remains unaffected, and the fiber effectively collects the light even after 100 welding tests (total welding time of 800 seconds). In addition, a more specific fiber treatment, in terms of the fiber protection removal, fiber cutting and fiber insertion into the TIG torch, could avoid the occurrence of the deterioration mentioned above, at least up to some extent. Measurements of the fiber transmission after several welding processes have shown the appearance of some wavelength-dependent losses in the UV region due to the intense plasma radiation. However, although special solarization-resistant fibers could be used to avoid this phenomenon, the effect of these losses is not especially relevant, as the fibers employed in our tests have proved to be suitable for plasma spectroscopic analysis. By means of several welding tests, and the determination of the associated $T_{e}$ profiles, the proposed fiber sensor has been successfully validated as a valid approach for on-line quality assurance of arc-welding processes.

This work has been co-supported by the Science and Technology Ministry of the Spanish Government through the CICYT TEC2004-05936-C02-02/MIC and TEC2005-08218-C02-02 projects.

\section{References}

1. C. S. Wu, M. Ushio, and M. Tanaka, "Analysis of the TIG welding arc behaviour," Comput. Mater. Sci. 7, 308-314 (1997).

2. H. Luo, H. Zeng, L. Hu, X. Hu, and Z. Zhou, "Application of artificial neural network in laser welding defect diagnosis," J. Mater. Process. Technol. 170, 403-411 (2005).

3. L. Grad, J. Grum, I. Polajnar, and J. M. Slabe, "Feasibility study of acoustic signals for on-line monitoring in short circuit gas metal are welding," Int. J. Mach. Tools Manuf. 44, 555-561 (2004).

4. H. C. Wilke III, S. Kottilingam, R. H. Zee, and B. A. Chin,
"Infrared sensing techniques for penetration depth control of the submerged arc welding process," J. Mater. Process. Technol. 113, 228-233 (2001).

5. A. Al-Habaibeh and R. Parkin, "An autonomous low-cost infrared system for the on-line monitoring of manufacturing processes using novelty detection," Int. J. Adv. Manuf. Technol. 22, 249-258 (2003).

6. P. J. Li and M. Zhang, "Analysis of an arc light mechanism and its application in sensing of the GTAW process," Weld. J. 79, 252-260 (2000).

7. A. Ancona, V. Spagnolo, P. M. Lugara, and M. Ferrara, “Optical sensor for real-time monitoring of $\mathrm{CO}_{2}$ laser welding process," Appl. Opt. 40, 6019-6025 (2001).

8. A. Cobo, F. Bardin, J. Mirapeix, D. P. Hand, J. D. C. Jones, and J. M. López-Higuera, "Optoelectronic device for non-invasive focal point measurement and control of the laser welding process," Meas. Sci. Technol. 16, N1-N6 (2005).

9. B. Sung-Hoon, K. Min-Suk, P. Seong-Kyu, C. Chin-Man, K. Cheol-Jung, and K. Kwang-Jung, "Auto-focus control and weld process monitoring of laser welding using chromatic filtering of thermal radiation," Meas. Sci. Technol. 11, 1772-1777 (2000).

10. F. M. Haran, D. P. Hand, C. Peters, and J. D. C. Jones, "Focus control system for laser welding," Appl. Opt. 36, 5246-5251 (1997).

11. H. R. Griem, Principles of Plasma Spectroscopy (Cambridge University Press, 1997).

12. W. Lochte-Holtgreven, Plasma Diagnostics (North-Holland, 1968).

13. A. Ancona, P. M. Lugara, F. Ottonelli, and I. M. Catalano, "A sensing torch for the on-line monitoring of the gas tungsten arc welding process of steel pipes," Meas. Sci. Tech. 15, 2412-2418 (2004).

14. L. L. Blyler, F. V. DiMarcello, J. R. Simpson, E. A. Sigety, A. C. Hart, and V. A. Foertmeyer, "UV-radiation induced losses in optical fibers and their control," J. Non-Cryst. Solids 38-39, 165-170 (1980).

15. J. Gombert, M. Ziegler, J. Assmus, K. F. Klein, G. Nelson, J. Clarkin, H. Pross, and J. Kiefer, "Radiation-damage of allsilica fibers in the UV-region," Proc. SPIE 3596, 124-132 (1999). 